\title{
Adiabatic expansion of nuclear matter gas to describe violation of Feynman scaling law in multiple particle production
}

\author{
Akinori Ohsawa ${ }^{1, *}$, Edison H. Shibuya ${ }^{2, * *}$, and Masanobu Tamada ${ }^{3, * * *}$ \\ ${ }^{1}$ Institute for Cosmic Ray Research, University of Tokyo. \\ ${ }^{2}$ Instituto de Fisica Gleb Wataghin, Universidade Estadual de Campinas. \\ ${ }^{3}$ Faculty of Science and Engineering, Kinki University.
}

\begin{abstract}
The main features of the rapidity density distribution of the produced hadrons in multiple particle production in nucleon collisions are; (a) the distribution in the forward region $(y \geq 0)$ has a shape similar to the Fermi distribution, (b) the distribution in the most forward region reaches almost the maximum rapidity $y_{\max }=\ln (\sqrt{s} / M)(M:$ nucleon mass $)$, and (c) the shrinkage of the distribution from the maximum rapidity increases with the incident energy (i.e. violation of Feynman scaling law).

These features are possible to be described by the assumptions that; (1) a fireball of the gas (made of nuclear matter, with the temperature $T_{i}$ and with the shape of the incident nucleon with Lorentz contraction) is produced in the collision, (2) the fireball makes the adiabatic expansion, and (3) the constituent particles of the gas obey the Maxwell-Boltzmann distribution of the temperature $T_{f}$ in the final state.
\end{abstract}

\section{Introduction}

In analyzing the data of multiple particle production in nucleon ( $p p$ and $\bar{p} p$ ) collisions, we assumed in our previous paper Ref. [1] a mechanism that several emitting centers are aligned on the rapidity axis and that each of them emits several hadrons of mainly pions, which will be called the emitting center model. The model contains only four parameters, the values of which are determined by fitting the resultant distributions of $p_{T}$ (the transverse momentum) and the rapidity density to those of the experiments at the incident energy $\sqrt{s}=22.4 \sim 7000 \mathrm{GeV}$. The model reproduces the data quite well.

In the model the emitting centers are distributed on the rapidity axis between $y=-y_{0} \sim y_{0}$. The distribution of them is of a flat shape with slant cuts at both shoulders, which was obtained by trial and error. The parameter $y_{0}$ is determined as $y_{0}=\ln \left(\sqrt{s} / M a_{2}\right)(M$ : nucleon mass), where $a_{2}=0.940(\sqrt{s} / 10 \mathrm{GeV})^{\alpha}$ with $\alpha=0.23$ by the experimental data, assuming the average inelasticity $<K>=0.5$. It indicates that the rapidity density distribution of the produced hadrons in the most forward region shrinks from the maximum rapidity $y_{\max }=\ln (\sqrt{s} / M)$ by a factor $\ln a_{2}\left(a_{2}>1\right)$ and that the shrinkage increases with the incident energy, i.e. violation of Feynman scaling law.

In this paper we propose possible physical processes to describe the violation of Feynman scaling law, which was obtained empirically in Ref. [1].

\footnotetext{
*e-mail: ohsawa@icrr.u-tokyo.ac.jp

**e-mail: ehiros@hotmail.com

***e-mail: msnbtamada@gmail.com
}

\section{Basic idea}

All discussions in the present paper are made in the center of mass system.

In the nucleon collision a fire-ball is produced at the collision point through the deposited energy by nucleons. The fire-ball is at rest at the collision point, and the shape and size of the fire-ball are the same as those of the incident nucleon with Lorentz contraction.

The fire-ball is made of a gas of high temperature, and the constituent particles of the gas are seeds of the hadrons which are produced in the collision.

The fire-ball expands along the collision axis approximately since the expansion occurs normally to the surface of the fireball with Lorentz contraction. The expansion is approximated to be one dimensional along the collision axis ( $z$-axis) for the sake of simplicity, since the longitudinal momentum is much larger than the transverse momentum for most of the constituent particles.

The temperature of the gas decreases through the expansion and the constituent particles transmute into the produced hadrons of mainly pions (hadronization) when the density of the constituent particles of the gas arrives at a certain fixed value. It is assumed that the rapidity of the produced hadron is the same as that of the constituent particle in the final state.

The following two points are assumed for the gas. (1) The temperature of the gas in the initial state is $T_{i}$, the energy dependence of which is is discussed in Sect. 4.

(2) The expansion of the gas is adiabatic.

The temperature $T$ of the gas is $T \propto 1 / V^{K-1}$ in the adiabatic expansion, where $\kappa$ and $V$ are the specific heat ratio and the volume of the gas, respectively. Hence the 
temperature of the gas at hadronization $T_{f}$ is

$$
\frac{T_{f}}{T_{i}}=\left[\frac{\frac{4}{3} \pi R_{0}^{3} / \gamma}{\pi R_{0}^{2} \times L}\right]^{(\kappa-1)}
$$

where $R_{0}$ and $\gamma$ are the radius of nucleon and the Lorentz factor of the incident nucleon, i.e. $\sqrt{s}=2 M \gamma$. The factor $L$ is the full length of the gas (of a cylindrical shape) along $z$ axis in the final state (at hadronization) and is defined as $L \equiv(4 / 3) R_{0} b$. That is,

$$
T_{f}=\frac{T_{i}}{(\gamma b)^{\kappa-1}}
$$

It is important to note that the temperature $T_{f}$ of the gas in the final state is dependent on the incident energy. The temperature $T_{f}$ concerns only the longitudinal momenta of the constituent particles, since the expansion of the constituent particles is approximated to be one dimensional.

\section{Rapidity density distribution}

The constituent particles are assumed to obey MaxwellBoltzmann distribution of the temperature $T_{f}$ in the final state. The one dimensional distribution is

$$
N^{\prime} \exp \left[-\frac{p_{z}^{2}}{2 m k T_{f}}\right] d p_{z} \quad\left(p_{z}=-\infty \sim \infty\right)
$$

by integrating $x$ and $y$ directions. Since the energy of the constituent particle is $\epsilon=p_{z}^{2} / 2 m$, it turns to

$$
\begin{aligned}
2 N^{\prime} e^{-\epsilon / k T_{f}} d p_{z} & \left(p_{z}=0 \sim \infty\right) \\
& =2 N^{\prime} e^{-\epsilon / k T_{f}} \frac{m d \epsilon}{p_{z}}
\end{aligned}
$$

If the above formula eq.(3) is valid in the relativistic energy region, too, the rapidity density distribution of the constituent particles is proportional to

$$
2 N^{\prime} m e^{-\mu^{\prime} \cosh (y) / k T_{f}} d y
$$

using the relations $\epsilon=\mu^{\prime} \cosh (y), p_{\|}=p_{z}=\mu^{\prime} \sinh (y)(y$ : rapidity) and $\mu^{\prime}=\sqrt{p_{T}^{\prime 2}+m^{\prime 2}}$ for the constituent particle.

Since the rapidity of the constituent particle is assumed to be the same as that of the produced hadron, the rapidity density distribution of charged produced hadrons is

$$
\frac{d N_{c h}}{d y}=A N e^{-\mu \cosh (y) / k T_{f}} \quad(y=0 \sim \infty)
$$

where $\mu=\sqrt{p_{T}^{2}+m^{2}}$ is for the produced pion (assuming that all produced hadrons are pions). The distribution of eq.(4) is normalized to $A$.

If the substantial range of the distribution is between $y=0 \sim y^{\prime}$ in eq.(4), the rapidity $y^{\prime}$ satisfies

$$
\frac{\mu \cosh \left(y^{\prime}\right)}{k T_{f}} \sim 1
$$

With the approximation of $\cosh \left(y^{\prime}\right) \cong e^{y^{\prime}} / 2\left(N . B . y^{\prime} \gg 1\right)$, the rapidity $y^{\prime}$ is

$$
y^{\prime}=\ln \left[\frac{2 k T_{f}}{\mu}\right]=\ln \left[\frac{2 k T_{i}}{\mu(b \gamma)^{K-1}}\right]=\ln \left[\frac{\sqrt{s}}{M} \frac{k T_{i}}{\gamma \mu(b \gamma)^{K-1}}\right]
$$

The last equation corresponds to the fact that the most forward region of the rapidity density distribution shrinks from the maximum rapidity $y_{\max }=\ln (\sqrt{s} / M)$. With the parameter $a$, defined as

$$
a \equiv \frac{\gamma \mu(b \gamma)^{\kappa-1}}{k T_{i}}=\frac{\gamma \mu}{k T_{f}}
$$

the rapidity $y^{\prime}$ is $y^{\prime}=\ln (\sqrt{s} / M a)$, which has the same form as the parameter $y_{0}=\ln \left(\sqrt{s} / M a_{2}\right)$ in Ref. [1]. Notice that the power-law energy dependence of the parameter $a_{2}$, which is determined empirically in Ref. [1], is also reproduced in the parameter $a$ owing to the adiabatic expansion of the gas and to the Lorentz contraction of the incident nucleon. It is worthy to mention that the values of the parameters $a$ and $a_{2}$ may be different by a factor since the definitions of them are different. That is, the former is for the produced hadrons, while the latter is for the emitting centers to emit the produced hadrons.

\section{Energy dependence of the parameter a}

The energy dependence of the parameter $a$ is determined by fitting the rapidity density distribution of eq.(4) to the experimental data of the pseudo-rapidity density. Both distributions of the rapidity and the pseudo-rapidity density are the same approximately since the relation $p_{T}^{2} \gg m^{2}$ ( $m$ : pion mass) holds barely. (The average value of $p_{T}$ by the experimental data is $\left\langle p_{T}\right\rangle=0.37 \sim 0.55 \mathrm{GeV} / c$ in the energy region of our concern.) The rapidity density at $y=0$ is replaced also by the pseudo-rapidity density at $\eta=0, \rho_{0}$. It is obtained reliably in Ref. [1] and is tabulated in Table 1 at various incident energies. That is,

$$
\frac{d N_{c h}}{d y} \cong \rho_{0} e^{-(2 M a / \sqrt{s})[\cosh (y)-1]}
$$

Figs. 1 and 2 show examples of the rapidity density distribution of eq.(6) and the pseudo-rapidity density distribution by the experiment. The values of the parameter $a$, obtained by fitting, are tabulated in Table 1 .

There is a small discrepancy between the curves and the data near $y=0$ due to the difference between the rapidity and the pseudo-rapidity density. (The former distribution is flat near $y=0$ while the latter has a shallow dip around $\eta=0$ with the center of the dip at $\eta=0$.)

Fig. 3 shows the energy dependence of the parameter $a$ in Table 1 . The solid line in Fig. 3 is approximated as

$$
a=1.09\left(\frac{\sqrt{s}}{10 \mathrm{GeV}}\right)^{0.37}=0.47\left(\frac{\sqrt{s}}{M}\right)^{0.37}
$$

In order to relate eq.(5) with the parameter $a$ of eq.(7), one needs the value of $\mu=\sqrt{p_{T}^{2}+m^{2}}$ for the produced pions. Since the relation $p_{T}^{2} \gg m^{2}$ holds as was discussed 


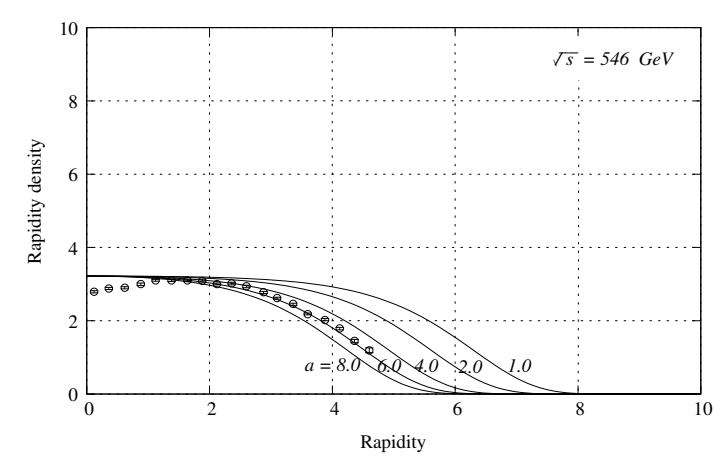

Figure 1. Rapidity density distribution at $\sqrt{s}=546 \mathrm{GeV}$. Lines are eq.(6) for $a=1.0,2.0,4.0,6.0$ and 8.0. Experimental data are the pseudo-rapidity density from UA5 experiment.

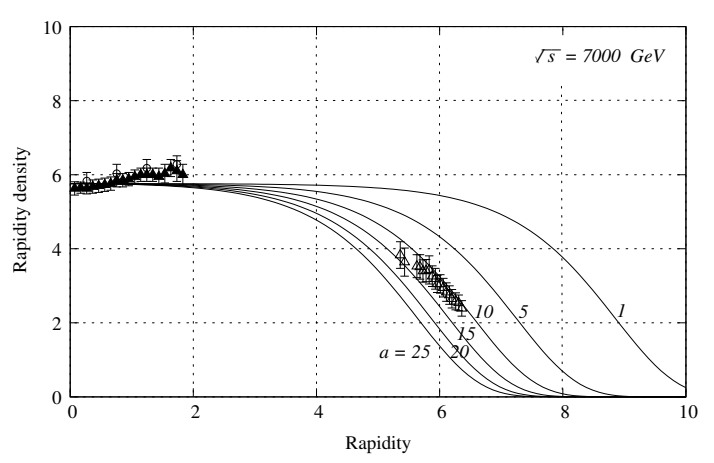

Figure 2. Rapidity density distribution at $\sqrt{s}=7000 \mathrm{GeV}$. Lines are eq.(6) for $a=1.0,5.0,10.0,15.0,20.0$ and 25.0. Experimental data in the most forward region are the pseudo-rapidity density from TOTEM (open triangles). Those in the central region are from ATLAS (full triangles) and CMS (open circles) experiments.

Table 1. Pseudo-rapidity density at $\eta=0$ and value of the parameter $a$.

\begin{tabular}{ccccc}
\hline$\sqrt{s}(\mathrm{GeV})$ & Exp. Group & $\rho_{0}{ }^{* *}$ & $a$ & Ref. \\
\hline 53 & UA5 & 1.92 & 2.0 & {$[2]$} \\
200 & UA5 & 2.54 & 3.0 & \\
200 & PHOBOS & 2.54 & 2.5 & {$[3]$} \\
546 & UA5 & 3.22 & 6.0 & \\
630 & UA7 & 3.27 & 6.0 & {$[4]$} \\
630 & P238 & 3.27 & 4.0 & {$[5]$} \\
630 & CDF & 3.27 & $* * *$ & {$[6]$} \\
900 & UA5 & 3.61 & 8.0 & \\
1800 & CDF & 4.23 & $* * *$ & \\
7000 & T.C.A & 5.76 & 12.0 & {$[7]$} \\
\hline${ }^{*}$ TOTEM, CMS, ATLAS, ${ }^{* *} \rho_{0}=\left(d N_{c h} / d \eta\right)_{\eta=0}$ \\
${ }^{* * *}$ No data in the forward region.
\end{tabular}

above, we have $\mu \cong p_{T}$ where $p_{T}$ is replaced by the average $<p_{T}>$. According to Ref.[1], the average $<p_{T}>$ of the produced hadrons by the experiments is

$$
<p_{T}>=0.33\left(\frac{\sqrt{s}}{10 \mathrm{GeV}}\right)^{0.08}=0.28\left(\frac{\sqrt{s}}{M}\right)^{0.08}(\mathrm{GeV} / c)
$$

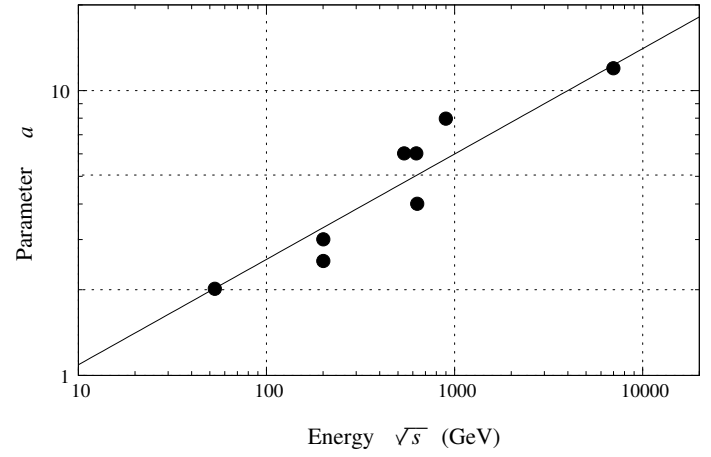

Figure 3. Energy dependence of the parameter $a$, tabulated in Table 1. The solid line is eq.(7) in the text.

Then eq.(5) turns to

$$
\begin{aligned}
& (b \gamma)^{k-1}=\frac{a k T_{i}}{\gamma \mu} \simeq \frac{a k T_{i}}{<p_{T}>\gamma} \\
& =\frac{0.47 \times 2}{0.28}\left(\frac{\sqrt{s}}{M}\right)^{0.37-0.08-1} \frac{k T_{i}}{\mathrm{GeV}}
\end{aligned}
$$

The parameter $b$ in eq.(9) is assumed to be proportional to the multiplicity $m_{c h}$, i.e. $b \equiv b_{0} m_{c h}$, where the charged multiplicity $m_{c h}$ by the experiments is approximated as

$$
m_{c h}=6.9\left(\frac{\sqrt{s}}{10 \mathrm{GeV}}\right)^{0.37}=2.9\left(\frac{\sqrt{s}}{M}\right)^{0.37}
$$

(See Ref. [1]). The assumption assures that the density of the constituent particles in the final state is fixed and independent of the incident energy. (See the discussion in Sect. 5.) Then eq.(9) turns to

$$
\left[\frac{2.9 b_{0}}{2}\left(\frac{\sqrt{s}}{M}\right)^{1+0.37}\right]^{K-1}=3.36\left(\frac{\sqrt{s}}{M}\right)^{0.29-1} \frac{k T_{i}}{\mathrm{GeV}}
$$

Assuming that the initial temperature is proportional to the incident energy, i.e. $T_{i} \propto \sqrt{s} / M$, the relation of the exponent is $1.37(\kappa-1)=0.29$, which leads to $\kappa=1.21$.

\section{Discussions}

(i) In the present paper we proposed a possible physical mechanism to describe the features of the rapidity density distribution of produced hadrons which is one of the important data of multiple particle production in nucleon collisions.

Main features of the rapidity density distribution are; (a) the distribution is symmetrical to the forward and the backward region and that of the forward region has a shape similar to Fermi distribution,

(b) the distribution extends almost to the maximum rapidity $y_{\max }=\ln (\sqrt{s} / M)$, that is, the longitudinal momentum $p_{\|}$is dependent on the incident energy (while $p_{T}$ is not approximately ${ }^{1}$ ), and

(c) the shrinkage of the distribution from the maximum

\footnotetext{
${ }^{1}$ The average of $p_{T}$ is dependent on the incident energy weakly. (See eq.(8) in the text.)
} 
rapidity increases with the incident energy, i.e. violation of Feynman scaling law.

Following physical processes (and assumptions) describe the features of the rapidity density distribution;

(1) in nucleon collision a fire-ball of a gas is formed at the collision point,

(2) the shape and size of the fire-ball in the initial state are the same as those of the incident nucleon with Lorentz contraction,

(3) the expansion of the gas is one dimensional and adiabatic.

(4) the constituent particles of the gas transmute into the produced hadrons at hadronization (The rapidity of the produced hadron is assumed to be equal to that of the constituent particle in the final state.),

(5) the full length of the gas of the cylinder shape in the final state is proportional to the multiplicity of the constituent particles,

(6) the constituent particles have momenta to obey the Maxwell-Boltzmann distribution in the final state, and

(7) eq.(3) is valid in the relativistic energy region.

In the present scheme the rapidity density distribution of produced hadrons has a simple form of

$$
e^{-\mu \cosh (y) / k T_{f}}=e^{-(2 M a / \sqrt{s}) \cosh (y)}
$$

( $T_{f}:$ the temperature of the gas in the final state), which reproduces the experimental data well by adjusting the value of the parameter $a$.

(ii) In the present framework the gas of the constituent particles has the specific heat ratio of $\kappa=1.21$, if we assume the temperature of the gas in the initial state is proportional to the incident energy. The value is near 1.29 and 1.30 of the gases, made of $\mathrm{CO}_{2}$ and $\mathrm{CH}_{4}$, respectively.

(iii) The full length of the gas in the final state increases with the incident energy, i.e. $L=(4 / 3) b_{0} R_{0} m_{c h}$ where the charged multiplicity $m_{c h}$ has the energy dependence. For example, $L=2.1 R_{0}$ and $5.0 R_{0}$ at $\sqrt{s}=100$ and $1000 \mathrm{GeV}$, respectively, for the assumed value of $b_{0}=0.1$. (There is no clue to infer the value of the parameter $b_{0}$.)
The number density of the constituent particles in the gas is

$$
n_{f}=\frac{(3 / 2) m_{c h}}{\pi R_{0}^{2} L}=\frac{9}{8 \pi R_{0}^{3} b_{0}}
$$

in the final state (at hadronization), since the number of the constituent particles is assumed to be equal to that of the produced hadrons. The density is $n_{f}=3.6 / R_{0}^{3}=$ $15.0 /\left(\frac{4}{3} \pi R_{0}^{3}\right)$ for $b_{0}=0.1$.

(iv) The derivation from eq.(2) to eq.(3) is tricky. However the resultant rapidity density distribution has a reasonable form and reproduces the experimental data well. ${ }^{2}$

(v) It is a merit of the emitting center model that it describes smoothly the facts that the longitudinal momentum $p_{\|}$of produced hadron is much larger than $p_{T}$ except the region near $y=0$ and that $p_{T}$, possible to be introduced in the scheme smoothly, is almost independent of the incident energy. Considering that the jets, observed in $e^{+} e^{-}$collisions, have similar characteristics to the emitting centers, the jets may be identified as the emitting centers, too. ${ }^{3}$

However the formation mechanism of the emitting centers has not been discussed physically but assumed. Present scheme is applicable to the emitting centers, too, instead of the constituent particles of the gas, which will be studied.

\section{References}

[1] A. Ohsawa, E.H. Shibuya, M. Tamada, Intern, Journ. of Mod. Phys. A27 No.9 1250043 (2012).

[2] J.G. Alner et al., Nucl. Phys. B291 261 (1987), and Phys. Rep. $1545 \& 6247$ (1987).

[3] B.B. Back et al., J. Phys. G: Nucl. Part. Phys. G30 S1133 (2004).

[4] E. Pare et al., Phys. Lett. B242 521 (1990).

[5] R. Haar et al., Phys. Lett. B401 176 (1997).

[6] F. Abe et al., Phys. Rev. D41 2330 (1990).

[7] G. Antchev et al. (TOTEM Collaboration), EPL 98 31002 (2012).

[8] H. Kumano, Prog. Theor. Phys. Suppl. No.76 51 (1983).

\footnotetext{
${ }^{2}$ The pseudo-rapidity density distribution by the experiments shows a shallow dip around $\eta=0$ with the center of the dip at $\eta=0$, which indicates that the rapidity density distribution is flat near $y=0$.

${ }^{3} \mathrm{~A}$ jet emits several hadrons and the $p_{T}$ distribution of the produced hadrons in the jet is similar to that in multiple particle production.[8]
} 